\title{
Some Remarks on Exp-Function Method and Its Applications
}

\author{
İsmail Aslan ${ }^{1, *}$ and Vangelis Marinakis ${ }^{2}$ \\ ${ }^{1}$ Department of Mathematics, İzmir Institute of Technology, Urla, İzmir 35430, Turkey \\ ${ }^{2}$ Department of Civil Engineering, Technological and Educational Institute of Patras, 1 M. Alexandrou Street, Koukouli, \\ 26334 Patras, Greece
}

(Received April 15, 2011; revised manuscript received June 17, 2011)

\begin{abstract}
Recently, many important nonlinear partial differential equations arising in the applied physical and mathematical sciences have been tackled by a popular approach, the so-called Exp-function method. In this paper, we present some shortcomings of this method by analyzing the results of recently published papers. We also discuss the possible improvement of the effectiveness of the method.
\end{abstract}

PACS numbers: 02.30.Jr, 04.20.Jb

Key words: Exp-function method, partial differential equations

\section{Introduction}

Exact and explicit solutions of nonlinear evolution equations (NEEs) may well describe distinct phenomena in the applied physical and mathematical sciences, as well as other fields. By means of these solutions, scientists may give better insight into the physical aspects of the nonlinear problems considered. During the past four decades or so, searching for analytic solutions of NEEs has been the main goal of many researchers. On this direction, a lot of powerful tools have been established and developed. To make mention of some, the homogeneous balance method, ${ }^{[1]}$ the inverse scattering, ${ }^{[2]}$ the Hirota's bilinear method, ${ }^{[3]}$ the Backlund transformation, ${ }^{[4]}$ the Painleve analysis, ${ }^{[5]}$ the symmetry approach, ${ }^{[6]}$ the F-expansion method, ${ }^{[7-8]}$ the sine-cosine method, ${ }^{[9]}$ the tanh-coth method, ${ }^{[10]}$ the homotopy perturbation, ${ }^{[11]}$ the Jacobi elliptic-function method, ${ }^{[12-13]}$ the extended tanhfunction method, ${ }^{[14]}$ and the similarity transformation method. ${ }^{[15-16]}$

Many of these methods take advantage of the availability of computer algebra systems (such as MATHEMATICA, MAPLE, and MATLAB), which avoid the need for doing the complex and tedious calculations "by hand" and eliminate error. In $2006, \mathrm{He}$ and $\mathrm{Wu}^{[17]}$ introduced such a method, the so-called Exp-function method, which relies on an ansatz (a rational combination of exponential functions), involving many unknown parameters to be specified at the stage of solving the problem. The method soon drew the attention of many researchers, who described it as "straightforward", "reliable", and "effective". Nowadays, being very popular, some authors put forth new modifications of the Exp-function method to tackle various kinds of nonlinear problems; just to mention a few, multi-dimensional equations, ${ }^{[18-19]}$ coupled NEEs, ${ }^{[20]}$ differential-difference equations, ${ }^{[21]}$ stochastic equations, ${ }^{[22]} \mathrm{NEEs}$ with variable coefficients, ${ }^{[23]} n$-soliton solutions, ${ }^{[24-27]}$ a multiple-exp function method, ${ }^{[28]}$ rational solutions, ${ }^{[29]}$ and a general $\operatorname{Exp}_{a}$-function method. ${ }^{[30]}$

On the other hand, Kudryashov ${ }^{[31-36]}$ and Parkes $^{[37-39]}$ have been constantly alerting the scientific community for equivalent, disguised, and incorrect results. A number of cases have been demonstrated in the literature ${ }^{[40-44]}$ where solutions derived by the Exp-function method are misleading. Consequently, the method has received a lot of criticisms from different points of views. ${ }^{[36,45-47]}$

In this paper we outline some further observations on the Exp-function method and its applications. Our goal is neither to defend nor to criticize the method, but rather to show why one should be extra careful when applying this method, as well as to discuss the improvement of the effectiveness (if any) of the method. In Sec. 2 we review the procedure of the Exp-function method, while in Secs. 3-8 we analyze the results of some published papers. We end our discussion with some concluding remarks in Sec. 9.

\section{Exp-Function Method}

Suppose we are given a partial differential equation (PDE) for a function $u(x, t)$ in the form

$$
P\left(u, u_{t}, u_{x}, u_{t t}, u_{t x}, u_{x x}, \ldots\right)=0,
$$

where $P$ is a polynomial in its arguments. The main idea of the Exp-function method for solving Eq. (1) proceeds in the following steps:

Step 1 Look for traveling wave solutions of Eq. (1) by taking the wave transformation

$$
u(x, t)=U(\eta), \quad \eta=k x+w t,
$$

into account, where $k$ and $w$ are real nonzero constants.

Step 2 Substitution of Eq. (2) into the PDE (1) yields an ordinary differentail equation (ODE) for $U(\eta)$. If possible, integrate the resulting ODE term by term one or more times. This introduces one or more integration constants.

\footnotetext{
${ }^{*}$ E-mail: ismailaslan@iyte.edu.tr

(C) 2011 Chinese Physical Society and IOP Publishing Ltd
} 
Step 3 Introduce the ansatz

$$
U(\eta)=\frac{a_{c} \exp (c \eta)+\cdots+a_{-d} \exp (-\mathrm{d} \eta)}{b_{p} \exp (p \eta)+\cdots+b_{-q} \exp (-q \eta)} .
$$

Here $c, d, p$, and $q$ are positive integers (to be determined). The coefficients $a_{i}$ and $b_{j}$ are arbitrary real constants (to be specified).

Step 4 Determine the highest order nonlinear term and the linear term of highest order in the ODE from Step 2, and express them in terms of Eq. (3). Then, in the resulting terms, balance the highest order Exp-function to determine $c$ and $p$, and the lowest order Exp-function to determine $d$ and $q$.

Step 5 With $c, d, p$, and $q$ as determined in Step 4, substitution of Eq. (3) into the ODE from Step 2 yields an algebraic equation involving the integer powers of $\exp (\eta)$. Equating the coefficients of each power of $\exp (\eta)$ to zero results in a (highly nonlinear) system of algebraic equations for $a_{i}, b_{j}, k$, and $w$. If the original PDE contains some arbitrary parameters, these will, of course, also appear in the system.

Step 6 The original PDE has a solution in the form (3) if and only if there is a real nontrivial solution to the resulting system of algebraic equations, and using a computer can be very helpful.

Remark When using the Exp-function method, some authors prefer to set the integration constants (obtained in Step 2) equal to zero. A solution obtained in this way usually becomes less general than it could be. The reason is that omitting these integration constants may lead to the loss of some arbitrary constants in the solution function. This fact has been formulated as the third common error in Ref. [35].

\section{A Careless Application of Exp-Function Method Leads to Incorrect Solutions}

The authors of Ref. [48] investigated three nonlinear equations based on the ansatz

$$
u(\eta)=\frac{a_{1} \exp (\eta)+a_{0}+a_{-1} \exp (-\eta)}{\exp (\eta)+b_{0}+b_{-1} \exp (-\eta)}, \quad \eta=k x+w t .
$$

Unfortunately, the authors of Ref. [48] left the reader with the impression that their solutions were new, while they are not even correct. First, it is claimed in Ref. [48] that the Klein-Gordon equation

$$
u_{t t}-u_{x x}-u+u^{3}=0
$$

admits the solution

$$
u(x, t)=\frac{\exp \left(k x+\sqrt{k^{2}+2} t\right)-(1 / 4) b_{0}^{2} \exp \left(-\left(k x+\sqrt{k^{2}+2} t\right)\right)}{\exp \left(k x+\sqrt{k^{2}+2} t\right)+b_{0}+(1 / 4) b_{0}^{2} \exp \left(-\left(k x+\sqrt{k^{2}+2} t\right)\right)},
$$

where $k$ and $b_{0}$ remain arbitrary (formula (3.15) in and thus, Ref. [48]). By a careful inspection, we observe that the function (5) can be further simplified as

$$
u(x, t)=\frac{-b_{0} / 2+\exp \left(k x+\sqrt{k^{2}+2} t\right)}{b_{0} / 2+\exp \left(k x+\sqrt{k^{2}+2} t\right)} .
$$

However, the direct substitution of the expression (5) (or its simplifed form above) into Eq. (4) yields

$$
\frac{16 b_{0} \exp \left(k x+\sqrt{2+k^{2}} t\right)\left(b_{0}-2 \exp \left(k x+\sqrt{2+k^{2}} t\right)\right)}{\left(b_{0}+2 \exp \left(k x+\sqrt{2+k^{2}} t\right)\right)^{3}},
$$

which is not zero in the general case. Thus, the function (5) does not satisfy Eq. (4). Moreover, the expression (3.16) in Ref. [48] (which is a special case of Eq. (3.15) with $b_{0}=2$ ) cannot be a solution of Eq. (4) as well, since the term (6) does not vanish either.

On the other hand, the wave transformation (2) reduces Eq. (4) to the ODE

$$
\left(w^{2}-k^{2}\right) U^{\prime \prime}-U+U^{3}=0 .
$$

We observe that Eq. (7) is embedded in the more general equation

$$
U^{\prime \prime}=F(U) .
$$

In fact, Eq. (8) can be solved by a quadrature. Multiplying both sides of Eq. (8) by $U^{\prime}$ and integrating the resulting equation once, we obtain

$$
\frac{1}{2}\left(U^{\prime}\right)^{2}=\int F(U) \mathrm{d} U+C=G(U), \quad C=\text { const. },
$$

$$
\mp \frac{\mathrm{d} U}{\sqrt{2 G(U)}}=\mathrm{d} \eta,
$$

which is a first-order equation with separable variables. In fact, if $G(U)$ is a polynomial of third or fourth degree, then the relation (9) constitutes the definition of elliptic integrals. Thus, the general solution of Eq. (7) is written in terms of elliptic functions.

Second, the authors of Ref. [48] considered the BurgerFisher equation

$$
u_{t}+u u_{x}+u_{x x}+u(1-u)=0,
$$

and found the exact solution (formula (4.13) in Ref. [48])

$$
u(x, t)=1-\frac{k}{k+1} \exp \left(-\left(k x+\left(k^{2}-k-1\right) t\right)\right),
$$

where $k$ remains arbitrary. However, the direct substitution of the expression (11) into Eq. (10) yields

$$
-\frac{k^{2} \exp \left(-2 k x+2\left(1+k-k^{2}\right) t\right)}{1+k},
$$

which is not zero in the general case as well. Hence, the function (11) cannot be a solution of Eq. (10).

Indeed, using the extended tanh-function method ${ }^{[49]}$ for instance, we observe that Eq. (10) admits the solution

$$
u(x, t)=\frac{1}{2}+\frac{1}{2} \tanh \left(\frac{1}{4}\left(x-\frac{5}{2} t\right)\right) .
$$

Third, the authors of Ref. [48] studied the SharmaTasso-Olver equation

$$
u_{t}+\alpha\left(u^{3}\right)_{x}+\frac{3}{2} \alpha\left(u^{2}\right)_{x x}+\alpha u_{x x x}=0,
$$


where $\alpha$ is a nonzero constant and found two exact solutions, which are numbered as (5.15) and (5.18) in there. For example, the solution (5.15) is given by

$u(x, t)=\frac{-2 k b_{-1} \exp \left(-\left(k x-4 \alpha k^{2} t\right)\right)}{\exp \left(k x-4 \alpha k^{2} t\right)+b_{-1} \exp \left(-\left(k x-4 \alpha k^{2} t\right)\right)}$,

where $k$ and $b_{-1}$ remain arbitrary. However, the direct substitution of the expression (13) into Eq. (12) yields

$$
\frac{16(k-1) k^{3} \alpha b_{-1} \exp (2 k(x+4 k \alpha t))}{\left(\exp (2 k x)+b_{-1} \exp \left(8 k^{2} \alpha t\right)\right)^{2}},
$$

which is not zero in the general case. Hence, the function (13) does not satisfy Eq. (12). Similarly, it can be shown that the solution (5.18) in Ref. [48] does not satisfy Eq. (12). Moreover, the expressions (5.16) and (5.19) in Ref. [48] cannot be solutions as well, since they also do not satisfy Eq. (12).

In fact, using the transformation $u(x, t)=G_{x} / G$, $G=G(x, t)$, Eq. (12) can be transformed to the third order linear partial differential equation

$$
G_{t}+\alpha G_{x x x}=0 .
$$

Then, by means of the wave transformation (2), Eq. (14) can be transformed further to the constant coefficient third order linear ODE

$$
\alpha k^{3} G^{\prime \prime \prime}+w G^{\prime}=0
$$

where the prime denotes the derivative with respect to $\eta$. From the linear theory, the general solution of Eq. (15) is well known to us.

Regrettably, the same authors have presented more incorrect results in Ref. [50]. More specifically, they have obtained six incorrect solutions (relations (3.14)-(3.16) and (3.18)-(3.20) in Ref. [50]) for the $(2+1)$-dimensional breaking soliton equation, and three incorrect solutions (relations (4.11), (4.15), and (4.19) in Ref. [50]) for the modified Zakharov-Kuznetsov equation. Moreover, they have studied an incorrect form of the KonopelchenkoDubrovsky equation with incorrect solutions (relations (5.14)-(5.16) in Ref. [50]).

\section{A Single Case of Exp-Function Method may Lead to Equivalent Solutions}

The author of Ref. [51] studied the Korteweg-de Vries equation which reads as $u_{t}+6 u u_{x}+u_{x x x}=0$ by means of the ansatz (due to the Exp-function method)

$$
u(x, t)=\frac{R k^{2}\left[A_{3} \exp (3 \eta)+A_{2} \exp (2 \eta)+A_{1} \exp (\eta)+A_{0}+A_{-1} \exp (-\eta)\right]}{\left(\exp (\eta)+b_{0}+b_{-1} \exp (-\eta)\right)\left(a_{1} \exp (\eta)+a_{0}+a_{-1} \exp (-\eta)\right)^{2}}, \quad \eta=k x+\omega t .
$$

Using the single ansatz (16) the author derived three solutions which are formulated as (24), (25), and (26) in there. For example, the solutions (24) and (25) in Ref. [51] are given respectively as

$$
\begin{aligned}
& u(x, t)=\frac{4 k^{2} a_{0} a_{-1}}{4 a_{-1}^{2} \exp (-\eta)+4 a_{0} a_{-1}+a_{0}^{2} \exp (\eta)}, \quad \eta=k x-k^{3} t, \\
& u(x, t)=\frac{8 k^{2} a_{-1} a_{1}}{\left(a_{1} \exp (\eta)+a_{-1} \exp (-\eta)\right)^{2}}, \quad \eta=k x-4 k^{3} t .
\end{aligned}
$$

Unfortunately, the author of Ref. [51] left the reader with the impression that these solutions are distinct. However, by minimizing the number of the parameters involved, we can rewrite Eqs. (17) and (18) as

$$
\begin{aligned}
& u(x, t)=\frac{4 k^{2} a_{0} a_{-1}}{4 a_{-1}^{2} \exp (-\eta)+4 a_{0} a_{-1}+a_{0}^{2} \exp (\eta)}=\frac{2 C_{1} k^{2} \exp (\eta)}{\left(1+C_{1} \exp (\eta)\right)^{2}}, \quad \eta=k x-k^{3} t, \quad C_{1}=\frac{a_{0}}{2 a_{-1}}, \\
& u(x, t)=\frac{8 k^{2} a_{-1} a_{1}}{\left(a_{1} \exp (\eta)+a_{-1} \exp (-\eta)\right)^{2}}=\frac{8 C_{2} k^{2} \exp (2 \eta)}{\left(1+C_{2} \exp (2 \eta)\right)^{2}}, \quad \eta=k x-4 k^{3} t, \quad C_{2}=\frac{a_{1}}{a_{-1}} .
\end{aligned}
$$

Now, letting $k \rightarrow 2 k$ in Eq. (19) leads to Eq. (20). Following the same approach one can show that the remaining solution (26) in Ref. [51] is equivalent to both solutions (24) and (25) presented in there.

\section{Different Cases of Exp-Function Method may Lead to Equivalent Solutions}

The authors of Ref. [52] applied the Exp-function method to the Radhakrishnan, Kundu and Laskshmanan equation which reads as

$$
\mathrm{i} u_{z}+u_{t t}+\frac{\gamma_{2}}{3 \gamma_{1}}|u|^{2} u+\mathrm{i} \gamma_{1} u_{t t t}+\mathrm{i} \gamma_{2}\left(|u|^{2} u\right)_{t}=0
$$

Using the ansatz (3) for the case $(p=c=1, d=q=1)$, they obtained the solution

$$
u(x, t)=\frac{a_{1} \exp (\alpha z-\omega t)+a_{0}-\left[\left(a_{1} b_{0}+a_{0}\right)\left(-a_{0}+a_{1} b_{0}\right) / 4 a_{1}\right] \exp (-(\alpha z-\omega t))}{\exp (\alpha z-\omega t)+b_{0}+\left[\left(a_{1} b_{0}+a_{0}\right)\left(-a_{0}+a_{1} b_{0}\right) / 4 a_{1}^{2}\right] \exp (-(\alpha z-\omega t))} \times \exp (\mathrm{i}(k z-c t)),
$$

which is formulated as Eq. (40) in Ref. [52]. Then, using the ansatz (3) for the case $(p=c=2, d=q=2)$, they obtained the solution

$$
u(x, t)=\frac{a_{2} \exp (2(\alpha z-\omega t))+a_{0}-\left[\left(a_{2} b_{0}+a_{0}\right)\left(-a_{0}+a_{2} b_{0}\right) / 4 a_{2}\right] \exp (-(2(\alpha z-\omega t)))}{\exp (2(\alpha z-\omega t))+b_{0}+\left[\left(a_{2} b_{0}+a_{0}\right)\left(-a_{0}+a_{2} b_{0}\right) / 4 a_{2}^{2}\right] \exp (-(2(\alpha z-\omega t)))} \times \exp (\mathrm{i}(k z-c t)),
$$

which is formulated as Eq. (45) in Ref. [52]. Finally, using the ansatz (3) for the case $(p=c=3, d=q=3)$, they obtained the solution

$$
u(x, t)=\frac{a_{3} \exp (3(\alpha z-\omega t))+a_{0}-\left[\left(a_{3} b_{0}+a_{0}\right)\left(-a_{0}+a_{3} b_{0}\right) / 4 a_{3}\right] \exp (-(3(\alpha z-\omega t)))}{\exp (3(\alpha z-\omega t))+b_{0}+\left[\left(a_{3} b_{0}+a_{0}\right)\left(-a_{0}+a_{3} b_{0}\right) / 4 a_{3}^{2}\right] \exp (-(3(\alpha z-\omega t)))} \times \exp (\mathrm{i}(k z-c t)),
$$


which is formulated as Eq. (50) in Ref. [52]. However, by a close inspection, we observe that these solutions are equivalent. For example, since $\alpha$ and $\omega$ are arbitrary constants, letting $\alpha \rightarrow \alpha / 2$ and $\omega \rightarrow \omega / 2$ in Eq. (22) leads to Eq. (21). By the same manner, one can obtain Eq. (21) from Eq. (23). Unfortunately, the authors of Ref. [52] left the reader with the impression that the solutions presented above are different. It is also worth to mention here that a similar incident has occured in Ref. [53] as well, where the authors have obtained equivalent solutions for the cases $(p=c=1, d=q=1)$, and $(p=c=2, d=q=2)$.

\section{Some Cases of Exp-Function Method are Equivalent}

The authors of Ref. [54] considered the cases $(p=c=2, d=q=2)$ (the formula (5.56) in there) and $(p=c=3$, $d=q=1$ ) (the formula (5.75) in there), respectively,

$$
\begin{aligned}
& \psi(\xi)=\frac{a_{2} \exp (2 \xi)+a_{1} \exp (\xi)+a_{0}+a_{-1} \exp (-\xi)+a_{-2} \exp (-2 \xi)}{b_{2} \exp (2 \xi)+b_{1} \exp (\xi)+b_{0}+b_{-1} \exp (-\xi)+b_{-2} \exp (-2 \xi)}, \\
& \psi(\xi)=\frac{a_{3} \exp (3 \xi)+a_{2} \exp (2 \xi)+a_{1} \exp (\xi)+a_{0}+a_{-1} \exp (-\xi)}{b_{3} \exp (3 \xi)+b_{2} \exp (2 \xi)+b_{1} \exp (\xi)+b_{0}+b_{-1} \exp (-\xi)},
\end{aligned}
$$

for solving a generalized nonlinear Schrödinger equation which is stated as (1.1) in Ref. [54]. However, if we multiply both the numerator and the denominator of Eq. (24) by $\exp (\xi)$ we obtain an expression identical to Eq. (25). Unfortunately, the authors of Ref. [54] left the reader with the impression that their solutions based on the ansatze (24) and (25) are different. We can find the same misleading in Refd. [53] and [55] as well. The authors of Ref. [53] considered the equivalent cases $(p=c=2, d=q=2)$ and $(p=c=3, d=q=1)$ while the author of Ref. [55] also studied the equivalent cases $(p=c=2, d=q=1)$ and $(p=c=1, d=q=2)$ in a redundant manner.

In summary we note that the following cases are equivalent according to the ansatz (3):

$$
\begin{aligned}
& (p=c=2, d=q=1) \equiv(p=c=1, d=q=2), \\
& (p=c=3, d=q=1) \equiv(p=c=2, d=q=2) \\
& \quad \equiv(p=c=1, d=q=3), \\
& (p=c=4, d=q=1) \equiv(p=c=3, d=q=2) \\
& \quad \equiv(p=c=2, d=q=3) \equiv(p=c=1, d=q=4),
\end{aligned}
$$

and so forth. This fact has also been emphasized in Refs. [42] and [56].

\section{Balancing Procedure of Exp-Function Method Seems Redundant}

As far as we could verify through the open literature, performing Step 4 in Sec. 2 takes a lot of effort. We have witnessed that all applications of the Exp-function method always lead to the single case $p=c$ and $d=q$. This fact has been proved in Ref. [56] by taking the linear and nonlinear terms of highest order as $u^{(n)}$ and $u^{r} u^{(s)}(s<n)$, respectively, in the ODE obtained in Step 2. Thus, Steps 2 and 4 seem to be redundant and time consuming. We believe that taking Step 3 (with Step 1) into account only and assigning arbitrary values to the positive integers $p(=c)$ and $d(=q)$ will greatly reduce the procedure of the Exp-function method and make laborious calculations unnecessary. Recently, the author of Ref. [57] brought three additional nonlinear terms of the form $u^{\alpha},\left(u^{(s)}\right)^{\Omega}$, and $\left(u^{(s)}\right)^{\Omega} u^{\lambda}$ into discussion and made exactly the same argument as was done by the author of Ref. [56]. It is clear that one can choose a highest order linear term in the form $u^{(n)}$. However, one cannot construct a general form for the highest order nonlinear term because there are many possibilities other than the ones considered in Refs. [5657]. Namely, the authors of Refs. [56-57] took some special cases of the nonlinear term into account. Hence, the problem still remains open.

Alternatively, since there are actually infinitely many cases that have to be considered separately, one can use the following procedure: Assume that there are solutions of the form

$$
u(x, t)=\frac{\sum_{k=0}^{n} a_{k} \exp (k \xi)}{\sum_{k=0}^{n} b_{k} \exp (k \xi)}, \quad \xi=b(x-c t),
$$

and start with the case $n=1$. Of course, $a_{0}, b_{0}$ or $a_{1}$, $b_{1}$ must not vanish at the same time, since this leads to a constant solution. Then proceed with the case $n=2$. Now, $a_{0}, b_{0}$ or $a_{1}, b_{1}$ or $a_{2}, b_{2}$ should not vanish at the same time, since this leads to the same solutions with case $n=1$. Then proceed with the case $n=3$ with similar restrictions, and so on.

\section{Not Every Generalization of Exp-Function Method is Convenient}

The authors of Ref. [58] proposed the so-called doubleExp function method for obtaining two-soliton solutions. The method was also used in Ref. [59]. Unfortunately, the double-Exp function method does not seem to work, since all results of Refs. [58] and [59] are incorrect. For example, the authors of Ref. [59] considered the generalized Burgers equations

$$
\begin{aligned}
& u_{t}+a\left(u^{n}\right)_{x}+b u_{x x}=0, \\
& u_{t}+a\left(u^{-n}\right)_{x}+b u_{x x}=0,
\end{aligned}
$$

where $a, b(\neq 0)$, and $n(>1)$ are arbitrary constants. Using the transformation $u=v^{1 /(n-1)}$, they reduced Eq. (26) into the equation

$$
v v_{t}+a n v^{2} v_{x}+b\left(\left(\frac{1}{n-1}-1\right) v_{x}^{2}+v v_{x x}\right)=0 .
$$

Then, by means of the ansatz (due to the double-Exp function method) 


$$
v(x, t)=\frac{a_{1} \exp (\xi)+a_{2} \exp (-\xi)+a_{5}+a_{3} \exp (\eta)+a_{4} \exp (-\eta)}{k_{1} \exp (\xi)+k_{2} \exp (-\xi)+k_{5}+k_{3} \exp (\eta)+k_{4} \exp (-\eta)}, \quad \xi=c_{1} x+c_{2} t, \quad \eta=c_{3} x+c_{4} t,
$$

they solved Eq. (28). As a result, the authors of Ref. [59] claimed that Eq. (28) admits one-soliton solutions (relations (3.9) and (3.10) in Ref. [59]), as well as a two-soliton solution (relation (3.11) in Ref. [59]). For example, the solution (3.9) in there is given by

$$
u(x, t)=\left(\frac{-2 b c_{3}\left(a_{1}+a_{4}\right)}{a(n-1)\left(a_{1}+a_{4}\right)-2 b c_{3} k_{3} \exp \left(c_{3}\left(x+\left(2 b c_{3} /(n-1)\right) t\right)\right)}\right)^{1 /(n-1)} .
$$

This means that, via the transformation $u=v^{1 /(n-1)}$, Eq. (28) admits the solution

$$
v(x, t)=\frac{-2 b c_{3}\left(a_{1}+a_{4}\right)}{a(n-1)\left(a_{1}+a_{4}\right)-2 b c_{3} k_{3} \exp \left(c_{3}\left(x+\left(2 b c_{3} /(n-1)\right) t\right)\right)} .
$$

However, the direct substitution of Eq. (31) into Eq. (28) results in the expression

$$
-\frac{8 b^{4}\left(a_{1}+a_{4}\right)^{2} c_{3}^{5} k_{3}\left(a(n-1)^{2}\left(a_{1}+a_{4}\right)+2 b c_{3} k_{3} \exp \left(c_{3}\left(x+\left(2 b c_{3} t /(n-1)\right)\right)\right) \exp \left(c_{3}\left(x+\left(2 b c_{3} t /(n-1)\right)\right)\right)\right.}{(n-1)\left(a(n-1)\left(a_{1}+a_{4}\right)-2 b c_{3} k_{3} \exp \left(c_{3}\left(x+\left(2 b c_{3} t /(n-1)\right)\right)\right)^{4}\right.},
$$

which is not zero in the general case. Thus, the function (30) cannot be a solution of Eq. (26). By the same token, we have verified that the other solutions (3.10) and (3.11) presented in Ref. [59] do not satisfy Eq. (26) as well. The same comments can be made for the incorrect solutions of Eq. (27) which are numbered as (3.16), (3.17), and (3.19) in Ref. [59], since they can be obtained by simply replacing $n$ with $-n$ in relations (3.9)-(3.11) of Ref. [59].

It is worth to mention here that we can solve Eq. (28) via the Exp-function method as follows: First of all, we can take $a=b=1$ in Eq. (28) without loss of generality. Thus, instead of Eq. (28), we can study the equation

$$
v v_{t}+n v^{2} v_{x}+\left(\frac{1}{n-1}-1\right) v_{x}^{2}+v v_{x x}=0
$$

Moreover, observe that if $v(x, t)$ is a solution of Eq. (32), then $-v(-x, t)$ is also a solution of Eq. (32). Taking the last remark and the issues outlined in the previous section into account, we first assume a function of the form

$$
v(x, t)=\frac{a_{0}+a_{1} \exp (\xi)}{b_{0}+b_{1} \exp (\xi)}, \quad \xi=b(x-c t),
$$

and substituting in Eq. (32) we find that the equation admits the solution

$$
v(x, t)=\frac{b b_{0}}{(1-n)\left(b_{0}+b_{1} \exp (\xi)\right)}, \quad \xi=b\left(x+\frac{b t}{n-1}\right),
$$

where $b_{0}, b_{1}$, and $b$ remain arbitrary. Thus, by setting $b_{0}=b_{1} b_{2}$, we conclude with the one-wave solution

$$
v(x, t)=\frac{b b_{2}}{(1-n)\left(b_{2}+\exp (\xi)\right)}, \quad \xi=b\left(x+\frac{b t}{n-1}\right),
$$

where $b$ and $b_{2}$ remain arbitrary. Then, by assuming a function of the form

$$
v(x, t)=\frac{a_{0}+a_{1} \exp (\xi)+a_{2} \exp (2 \xi)}{b_{0}+b_{1} \exp (\xi)+b_{2} \exp (2 \xi)}, \quad \xi=b(x-c t)
$$

and substituting in Eq. (32) it is quite easy to verify that all solutions obtained are equivalent to Eq. (334). Of course, during the above process, we assume that $n \neq 2$, otherwise we have the classical Burgers equation which is known to be integrable. ${ }^{[60]}$

Regarding two-wave solutions, we attempted to solve Eq. (32) via the ansatz (29) but failed. We believe that the ansatz (29) is not convenient, since some intermediate constants are actually set equal to zero and some solutions may be missed. Alternatively, we followed Ref. [24] and searched for a two-wave solution of Eq. (32) in the form

$$
v(x, t)=\frac{a_{10} \exp \left(\xi_{1}\right)+a_{01} \exp \left(\xi_{2}\right)+a_{20} \exp \left(2 \xi_{1}\right)+a_{11} \exp \left(\xi_{1}+\xi_{2}\right)+a_{02} \exp \left(2 \xi_{2}\right)}{1+b_{10} \exp \left(\xi_{1}\right)+b_{01} \exp \left(\xi_{2}\right)+b_{20} \exp \left(2 \xi_{1}\right)+b_{11} \exp \left(\xi_{1}+\xi_{2}\right)+b_{02} \exp \left(2 \xi_{2}\right)},
$$

where $\xi_{i}=b_{i}\left(x-c_{i} t\right) i=1,2$. However, a first study of the resulting algebraic system led only to two cases:

(i) $n=2$, which corresponds to the classical Burgers equation.

(ii) $b_{1}=b_{2}$ and $c_{1}=c_{2}$, which turns Eq. (35) into a one-wave.

Thus, the problem of finding multi-wave solutions for Eqs. (26) and (27) remains open.

\section{Concluding Remarks}

The Exp-function method has initiated an explosive reaction in the scientific community to find supposedly new exact solutions of nonlinear evolution equations. It is very likely that the flow of papers describing the application of this method will continue in the future.

In this paper, unlike Ref. [35], we focus on some shortcomings of the Exp-function method only. First, we refer to two of the many papers in which the careless application leads to incorrect results. As mentioned in Step 5 of Sec. 2, substitution of Eq. (3) into Eq. (1) usually results in a highly nonlinear algebraic system. Thus, one encounters tedious calculations (even when using a computer algebra system) and must be extra careful not to make any mistakes, as well as not to miss any solution of the system, thus not to miss any solution of the equation. It would be wise always to check our results by direct substitution.

Secondly, we refer to some redundant calculations that should be omitted. Thus, the balancing procedure cannot 
add much and should be left aside, while some cases of the Exp-function method are identical, and should not be considered as well, since they lead to the same solutions. Moreover, we refer to some examples where a single case or different cases of the Exp-function method lead to equivalent solutions. Thus, after finding some solutions, one should not leave them as they are, but rather compare them with each other and/or try to simplify them, by omitting some redundant constants or else.

To summarize, we believe that there should never be a blind trust in using the Exp-function method, one should always be able to justify why the output is a believable answer.

Last, we concentrate on a particular work, where the so-called double-Exp function method is used in order to produce both one-soliton and two-soliton solutions. We first show that all the results are wrong and present the correct one-wave solution. Then we argue on the ansatz (29) and propose the use of a form (35), as being more convenient. In fact, it is easy to verify that the single form

$$
u(x, t)=\frac{f_{1}\left(\xi_{1}, \xi_{2}\right)}{f_{2}\left(\xi_{1}, \xi_{2}\right)}, \quad \xi_{i}=b_{i}\left(x-c_{i} t\right), \quad i=1,2,
$$

where

$$
f_{1}\left(\xi_{1}, \xi_{2}\right)=a_{10} \exp \left(\xi_{1}\right)+a_{01} \exp \left(\xi_{2}\right)+a_{20} \exp \left(2 \xi_{1}\right)
$$

$$
\begin{aligned}
& +a_{11} \exp \left(\xi_{1}+\xi_{2}\right)+a_{02} \exp \left(2 \xi_{2}\right) \\
& +a_{30} \exp \left(3 \xi_{1}\right)+a_{21} \exp \left(2 \xi_{1}+\xi_{2}\right) \\
& +a_{12} \exp \left(\xi_{1}+2 \xi_{2}\right)+a_{03} \exp \left(3 \xi_{2}\right) \\
f_{2}\left(\xi_{1}, \xi_{2}\right)= & 1+b_{10} \exp \left(\xi_{1}\right)+b_{01} \exp \left(\xi_{2}\right) \\
& +b_{20} \exp \left(2 \xi_{1}\right)+b_{11} \exp \left(\xi_{1}+\xi_{2}\right) \\
& +b_{02} \exp \left(2 \xi_{2}\right)+b_{30} \exp \left(3 \xi_{1}\right) \\
& +b_{21} \exp \left(2 \xi_{1}+\xi_{2}\right)+b_{12} \exp \left(\xi_{1}+2 \xi_{2}\right) \\
& +b_{03} \exp \left(3 \xi_{2}\right)+b_{40} \exp \left(4 \xi_{1}\right) \\
& +b_{31} \exp \left(3 \xi_{1}+\xi_{2}\right)+b_{22} \exp \left(2 \xi_{1}+2 \xi_{2}\right) \\
& +b_{13} \exp \left(\xi_{1}+3 \xi_{2}\right)+b_{04} \exp \left(4 \xi_{2}\right)
\end{aligned}
$$

can reveal in a very simple way the known two-wave solutions of many significant PDEs, such as the Burgers, the $\mathrm{KdV}$, the modified $\mathrm{KdV}$, the sine-Gordon, and the Fitzhugh-Nagumo equations.

There are of course many significant PDEs that admit rational-wave solutions, i.e., rational combinations of exponentials and polynomial functions. One such example is the Fitzhugh-Nagumo equation which reads as $u_{t}=u_{x x}+u(1-u)(u-a)$, and, for $a=1$, admits the solution

$$
u(x, t)=\frac{\sqrt{2}\left(c_{1} \sqrt{2}+2 c_{2}+c_{2} \sqrt{2} x+2 c_{2} t\right) \exp (x \sqrt{2} / 2-t / 2)}{2\left[c_{0}+\left(c_{1}+c_{2} x+\sqrt{2} c_{2} t\right) \exp (\sqrt{2} x / 2-t / 2)\right]},
$$

where $c_{0}, c_{1}$, and $c_{2}$ are arbitrary constants. ${ }^{[36]}$ Of course, the generalization (36) of the Exp-function method cannot reveal such solutions, due to the initially assumed form of the solution. However, we can alternatively generalize relation (33) by considering polynomials in $x$ and $t$, instead of constants. Thus, we can assume that

$$
u(x, t)=\frac{\left(a_{00}+a_{10} x+a_{01} t\right)+\left(a_{11}+a_{20} x+a_{02} t\right) \exp (\xi)}{\left(b_{00}+b_{10} x+b_{01} t\right)+\left(b_{11}+b_{20} x+b_{02} t\right) \exp (\xi)}, \quad \xi=b(x-c t) .
$$

Clearly, using the ansatz (38), we can now reveal solutions such as Eq. (37).

\section{Acknowledgments}

The authors would like to thank the referee for the valuable and useful suggestions.

\section{References}

[1] M.L. Wang, Phys. Lett. A. 213 (1996) 279.

[2] M.J. Ablowitz and H. Segur, Solitons and Inverse Scattering Transform, SIAM, Philadelphia (1981).

[3] R. Hirota, The Direct Method in Soliton Theory, Cambridge University Press, Cambridge (2004).

[4] R.M. Miura, Backlund Transformation, Springer-Verlag, New York (1973).

[5] J. Weiss, M. Tabor, and G. Carnevale, J. Math. Phys. 24 (1983) 522 .

[6] G.W. Bluman and S.Kumei, Symmetries and Differential Equations, Springer-Verlag, New York (1989).

[7] J.F. Zhang, C.Q. Dai, Q. Yang, and J.M. Zhu, Opt. Commun. 252 (2005) 408.

[8] Y.X. Chen and X.H. Lu, Commun. Theor. Phys. 55 (2011) 871

[9] C. Yan, Phys. Lett. A 224 (1996) 77.
[10] W. Malfliet and W. Hereman, Phys. Scr. 54 (1996) 563.

[11] J.H. He, Appl. Math. Comput. 151 (2004) 287.

[12] Z.T. Fu, Z.H. Yao, S.K. Liu, and S.D. Liu, Commun. Theor. Phys. 44 (2005) 23.

[13] C.Q. Dai and J.F. Zhang, Internat. J. Modern Phys. B 19 (2005) 2129.

[14] C.Q. Dai, Q. Yang, and J.F. Zhang, Z. Naturforsch. A 59 (2004) 635.

[15] C.Q. Dai, Y.Y. Wang, and J.F. Zhang, Opt. Lett. 35 (2010) 1437.

[16] C.Q. Dai, Y.Y. Wang, and C.J. Yan, Opt. Commun. 283 (2010) 1489.

[17] J.H. He and X.H. Wu, Chaos, Solitons \& Fractals 30 (2006) 700.

[18] I. Aslan, Appl. Math. Comput. 217 (2010) 1421.

[19] I. Aslan, Appl. Math. Comput. 217 (2011) 6013.

[20] E. Yomba, Phys. Lett. A 373 (2009) 4001. 
[21] S.D. Zhu, Int. J. Nonlinear Sci. Numer. Simul. 8 (2007) 461.

[22] C.Q. Dai and J.F. Zhang, Int. J. Nonlinear Sci. Numer. Simul. 10 (2009) 675.

[23] S. Zhang, Phys. Lett. A 365 (2007) 448.

[24] V. Marinakis, Z. Naturforsch. 63a (2008) 653.

[25] İ. Aslan, Math. Methods Appl. Sci. 34 (2011) 990.

[26] İ. Aslan, Math. Methods Appl. Sci., to appear.

[27] İ. Aslan, Int. J. Nonlinear Sci. Numer. Simul. 11 (2010) 619.

[28] W.X. Ma, T. Huang, and Y. Zhang, Phys. Scr. 82 (2010) 065003 (8pp).

[29] S. Zhang, Nonl. Sci. Lett. A 1 (2010) 143.

[30] A.T. Ali and E.R. Hassan, Appl. Math. Comput. 217 (2010) 451.

[31] N.A. Kudryashov and M.B. Soukharev, Regul. Chaotic Dyn. 14 (2009) 407.

[32] N.A. Kudryashov, Commun. Nonlinear Sci. Numer. Simul. 15 (2010) 2778.

[33] N.A. Kudryashov and M.B. Soukharev, Commun. Nonlinear Sci. Numer. Simul. 15 (2010) 1765.

[34] N.A. Kudryashov, Commun. Nonlinear Sci. Numer. Simul. 14 (2009) 1891.

[35] N.A. Kudryashov, Commun. Nonlinear Sci. Numer. Simul. 14 (2009) 3507.

[36] N.A. Kudryashov and N.B. Loguinova, Commun. Nonlinear Sci. Numer. Simul. 14 (2009) 1881.

[37] E.J. Parkes, Commun. Nonlinear Sci. Numer. Simul. 15 (2010) 2769.

[38] E.J. Parkes, Appl. Math. Comput. 215 (2009) 864.

[39] E.J. Parkes, Appl. Math. Comput. 217 (2010) 3575.

[40] N.A. Kudryashov, Phys. Lett. A 373 (2009) 1196.
[41] N.A. Kudryashov, J. Comput. Appl. Math. 234 (2010) 3511.

[42] I. Aslan, J. Comput. Appl. Math. 234 (2010) 3213.

[43] I. Aslan, Comput. Math. Appl. 61 (2011) 1700.

[44] I. Aslan, Appl. Math. Comput. 217 (2010) 2912.

[45] Z. Navickas and M. Ragulskis, Appl. Math. Comput. 211 (2009) 522.

[46] Z. Navickas, L. Bikulciene, and M. Ragulskis, Appl. Math. Comput. 216 (2010) 2380.

[47] Z. Navickas, M. Ragulskis, and L. Bikulciene, Commun. Nonlinear Sci. Numer. Simul. 15 (2010) 3874.

[48] A. Bekir and A. Boz, Phys. Lett. A 372 (2008) 1619.

[49] E. Fan, Phys. Lett. A 277 (2000) 212.

[50] A. Bekir and A. Boz, Chaos, Solitons \& Fractals 40 (2009) 458.

[51] C. Chun, Comput. Math. Appl. 61 (2011) 2107.

[52] D.D. Ganji, A. Asgari, and Z.Z. Ganji, Acta Appl. Math. 104 (2008) 201.

[53] B.C. Shin, M.T. Darvishi, and A. Barati, Comput. Math. Appl. 58 (2009) 2147.

[54] M.Y. Moghaddam, A. Asgari, and H. Yazdani, Appl. Math. Comput. 210 (2009) 422.

[55] E. Yusufoğlu, Phys. Lett. A 372 (2008) 442.

[56] A.T. Ali, Phys. Scr. 79 (2009) 025006.

[57] A. Ebaid, J. Math. Anal. Appl., to appear.

[58] H.M. Fu and Z.D. Dai, Int. J. Nonlinear Sci. Numer. Simul. 10 (2009) 927.

[59] J. Liu, G. Mu, Z. Dai, and X. Liu, Comput. Math. Appl. 61 (2011) 1995.

[60] P.G. Drazin and R.S. Johnson, Solitons: An Introduction, Cambridge University Press, Cambridge (1996). 